\title{
Ciclo da assistência farmacêutica e a atuação do farmacêutico em unidades básicas de saúde de Santa Maria - RS
}

\author{
Cycle of pharmaceutical assistance and practice of \\ pharmacist in basic health units at Santa Maria - RS
}

Francieli Barchet BISCAHYNO, Jane Beatriz LIMBERGER* Centro universitário Franciscano/ Curso de Farmácia, Rua dos Andradas, 1614 CEP 97010032. Santa Maria, RS E-mail: janebeatriz@unifra.br

\begin{abstract}
According to the Brazilian Consensus of pharmaceutical care, pharmaceutical care is a set of actions developed by the pharmacist, and other health professionals, focused on the promotion, protection and recovery of health, both individually and collectively. In this context, the professional practice of pharmaceutical services permeates the activities involved in the cycle of pharmaceutical care, aiming at continuous supply of quality medicines to patients. This study aimed to assess the reality of pharmaceutical care in Santa Maria through the identification of actors responsible for medicines in Basic Health Units (BHU) namely health professionals that has responsibility for actions related to the drug in these locations. It was evident the importance of the pharmacist for the qualification of the activities involved with the cycle of pharmaceutical care as well as to control and evaluation. The inclusion of pharmacists in health care teams is still a challenge to be searched and depends on the awareness of managers about the importance of this work for improving medication management and quality of life of patients.
\end{abstract}

KEYWORDS: Pharmaceutical care; Pharmacist; Health Basic Units

\section{RESUMO}

De acordo com o Consenso Brasileiro de Atenção Farmacêutica, a assistência farmacêutica é um conjunto de ações desenvolvidas pelo farmacêutico, e outros profissionais de saúde, voltadas à promoção, proteção e recuperação da saúde, tanto em nível individual como coletivo. Neste contexto, a atuação do profissional na assistência farmacêutica municipal perpassa as atividades envolvidas no ciclo da assistência farmacêutica, visando à contínua oferta de medicamentos de qualidade ao paciente. Este estudo visou avaliar a realidade da assistência farmacêutica no município de Santa Maria através da identificação de atores responsáveis pelos medicamentos nas Unidades Básicas de Saúde (UBSs) ou seja, dos profissionais da saúde que detém a responsabilidade pelas ações relativas ao medicamento nestes locais. Ficou evidente a importância do profissional farmacêutico para a qualificação das atividades envolvidas com o ciclo da assistência farmacêutica, bem como para seu controle e avaliação. A inclusão do profissional farmacêutico nas equipes de saúde ainda é um desafio a ser buscado e depende da conscientização dos gestores sobre a importância deste profissional para a melhoria da gestão de medicamentos e da qualidade de vida dos pacientes.

PALAVRAS CHAVE: Assistência farmacêutica; Farmacêutico; Unidades Básicas de Saúde. 
LISTA DE ABREVIAÇÕES:

ANVISA Agência Nacional de Vigilância Sanitária

BPF Boas Práticas de Fabricação

OMS Organização Mundial da Saúde

PNRS Política Nacional de Resíduos Sólidos

REMUME Relação Municipal de Medicamentos Essenciais

RENAME Relação Nacional de Medicamentos Essenciais

SUS Sistema Único de Saúde

UBS Unidade Básica de Saúde

\section{INTRODUÇÃO}

A assistência farmacêutica é um conjunto de ações desenvolvidas pelo farmacêutico, e outros profissionais de saúde, voltadas a promoção, proteção e recuperação da saúde, tanto a nível individual como coletivo, tendo o medicamento como insumo essencial e visando o acesso e o seu uso racional. Envolve a pesquisa, o desenvolvimento e a produção de medicamentos e insumos, bem como a sua seleção, programação, aquisição, distribuição, dispensação, garantia da qualidade dos produtos e serviços, acompanhamento e avaliação de sua utilização, na perspectiva da obtenção de resultados concretos e da melhoria da qualidade de vida da população (1).

Pode-se dizer que a assistência farmacêutica atua conciliando ações entre o medicamento e o seu usuário e possibilita voltar a atenção não somente ao medicamento, mas também ao indivíduo que dele faz uso, proporcionando a prevenção, identificação e resolução de problemas relacionados a medicamentos. A Organização Mundial de Saúde reconhece que o farmacêutico é profissional com melhor capacitação para conduzir as ações destinadas à melhoria do acesso e promoção do uso racional dos medicamentos, sendo ele indispensável para organizar os serviços de apoio necessários para o desenvolvimento pleno da assistência farmacêutica (2).

Uma das responsabilidades do farmacêutico é acompanhar o medicamento durante o tratamento junto ao paciente, para avaliar se os objetivos terapêuticos foram alcançados, para identificar possíveis efeitos adversos e analisar os resultados obtidos, pois não cabe a esse profissional aplicar seu conhecimento apenas para confirmar a boa escolha do medicamento, para dispensá-lo ou para manter sua conservação. Sem a atuação do farmacêutico os medicamentos e os cuidados relacionados ao seu uso correm o risco de ficar soltos, desordenados e desqualificados dentro das UBSs.

O acesso da população a uma assistência farmacêutica de qualidade representa um dos maiores desafios para os sistemas de saúde, principalmente nos países subdesenvolvidos. Neste sentido, avaliar as ações promovidas nas Unidades Básica de Saúde visando à adequação ao ciclo da assistência farmacêutica constitui-se em um passo primordial para a organização do serviço, pois permite que pontos positivos e negativos sejam levantados e soluções sejam apontadas para a melhoria do serviço, que em última instância, visa à melhoria da qualidade de vida da população no que tange ao uso de medicamentos.

Neste contexto, esta pesquisa teve como objetivo avaliar a adequação das atividades do farmacêutico ao ciclo da assistência farmacêutica em UBSs do município de Santa Maria, determinar o contexto da assistência farmacêutica nas unidades, demonstrar a importância da integração entre cada etapa do ciclo e relatar a relevância do profissional farmacêutico e da assistência farmacêutica no Sistema Único de Saúde (SUS).

\section{MATERIAIS E MÉTODOS}

Trata-se de uma pesquisa observacional descritiva, retrospectiva, transversal, visando avaliar a realidade da assistência farmacêutica no município de Santa Maria. A fonte primária de dados foi constituída por dois profissionais farmacêuticos responsáveis pelas farmácias das UBSs e outros dois profissionais, de formação distinta, que detém a responsabilidade pelas ações relativas ao medicamento em suas UBSs. O critério de escolha das UBSs considerou a equivalência bem como a área de abrangência e população atendida, tendo o cuidado de escolher unidades que apresentavam volume de demanda semelhante.

Este artigo contempla as normas preconizadas pela RE 196/96, no que diz respeito aos aspectos éticos da pesquisa com seres humanos, sendo aprovado pelo comitê de ética do Centro Universitário Franciscano - Unifra, sobre registro no CEP/UNIFRA 427.2010.2.

\section{RESULTADOS E DISCUSSÃO}

Primeiramente, buscou-se caracterizar os profissionais envolvidos com a assistência farmacêutica nas unidades básicas de saúde no que diz respeito à profissão e tempo de atividade. A partir deste critério foram selecionados dois Profissionais farmacêuticos (denominados $\mathrm{P} 1$ e P2) e dois profissionais enfermeiros (denominados P3 e P4). Este número de unidades foi selecionado em virtude de só haver, no município, dois farmacêuticos atuando em unidades básicas de saúde, e para fins de comparação, foram selecionadas duas outras unidades, de mesmo tamanho e região geográfica das unidades sob responsabilidade de um farmacêutico. 
Tabela 1. Conhecimento relativo à etapa de seleção do ciclo da assistência farmacêutica (P1 e P2 - Farmacêuticos; P3 e P4 - Enfermeiros). Santa Maria-RS, 2010.

\begin{tabular}{l|l|l|l|l} 
& \multicolumn{1}{c|}{ P1 } & \multicolumn{1}{c|}{ P2 } & \multicolumn{1}{c}{ P3 } & P4 \\
\hline $\begin{array}{l}\text { Quais critérios são } \\
\text { utilizados para compra } \\
\text { de medicamentos? }\end{array}$ & Rename & Registro na ANVISA, BPF. & Rename & Regime de preço \\
\hline $\begin{array}{l}\text { É utilizada DCB? } \\
\text { Quais critérios de orga- } \\
\text { nização são utilizados? }\end{array}$ & Ordem alfabética & Ordem alfabética & Ordem alfabética & $\begin{array}{l}\text { Por grupo terapêutico } \\
\text { sequencial }\end{array}$ \\
\hline
\end{tabular}

Em relação ao tempo de serviço, os farmacêuticos possuíam menor tempo de serviço (4 e 10 anos) em comparação aos outros profissionais (14 e 19 anos). O tempo de serviço pode ser um fator determinante para o conhecimento de práticas envolvidas nos processos da assistência farmacêutica, pois o contato constante com políticas e práticas acaba por familiarizar o trabalhador com os conceitos e aprimora suas atividades. Pode-se, porém, perceber que muitas vezes ações rotineiras realizadas há muito tempo tendem a ser banalizadas quando não se reconhece sua importância, desqualificando o resultado final.

Os participantes foram, então, questionados sobre as etapas do ciclo da assistência farmacêutica, a saber: seleção, programação, aquisição, armazenamento, distribuição e utilização de medicamentos. Teve-se por objetivo levantar o conhecimento sobre as práticas profissionais necessárias para a condução do processo.

A seleção de medicamentos, atividade mais importante do ciclo, (3) é o processo de escolha de medicamentos eficazes e seguros imprescindíveis ao atendimento das necessidades de uma dada população, tendo como base as doenças prevalentes (4). A seleção de medicamentos essenciais tende a incrementar a qualidade da prescrição e a facilitar o processo da escolha do medicamento e da dispensação. O abastecimento nas UBS, inclusive o armazenamento e a distribuição, também pode ser mais regular e eficiente, com economia e racionalização (5). As questões abordadas sobre essa etapa do ciclo podem ser visualizadas Tabela 1. Um dos farmacêuticos entrevistados fez parte e outro é membro da Comissão de Farmacoterapêutica do município, tendo, ambos, conhecimentos sobre critérios de seleção de medicamentos. Porém, um dos farmacêuticos citou como critérios de seleção o registro do produto na ANVISA e a exigência de Boas Práticas de Fabricação (BPF), que acreditamos ser uma confusão das etapas de seleção e aquisição. Fato semelhante aconteceu com os demais profissionais, uma vez que um deles indicou que o critério de seleção era baseado na Relação Nacional de Medicamentos Essenciais (RENAME) e outro que tinha relação com o Regime de Preços praticado, novamente demonstrando confusão de conceitos.

Todos os entrevistados tem acesso e conhecem a Relação Municipal de Medicamentos Essenciais (REMUME), admitindo a utilização da Nomenclatura Comum Brasileira (DCB) em seus itens e, exceto por uma profissional que acreditava que os medicamentos estavam listados por grupo terapêutico e dispostos em ordem alfabética.

A etapa seguinte diz respeito à programação dos medicamentos selecionados, que tem por objetivo a garantia da disponibilidade dos medicamentos previamente selecionados nas quantidades adequadas e no tempo oportuno para atender às necessidades de uma população-alvo. A programação é um dos pontos cruciais do ciclo da assistência farmacêutica por sua relação direta com o nível de acesso aos medicamentos e as perdas relacionadas aos mesmos (6). Os farmacêuticos reconhecem a existência de critérios de seleção, baseados na demanda, e descrevem o intervalo de programação da Unidade Básica como quinzenal. Um dos demais profissionais não soube responder quais critérios eram utilizados, porém, ambos souberam afirmar que a programação era quinzenal (Tabela 2). A aquisição é conjunto de procedimentos pelos quais se efetiva a compra de medicamentos, de acordo com uma programação estabelecida, com o objetivo de suprir necessidades de medicamentos em quantidade, qualidade e menor custo-efetividade e manter a regularidade do sistema (7). O processo de aquisição para ser bem executado, deve considerar diversos fatores e atender alguns requisitos, tais como: uma programação de medicamentos, a existência de uma 
política de aquisição definindo se a forma de compra é centralizada ou descentralizada, a fonte de recursos, normas e procedimentos operacionais com definição explícita das responsabilidades e fluxo operacional do processo de compra (8). A aquisição na administração pública ocorre por meio de processo licitatório que consiste em um procedimento administrativo preliminar mediante o qual a administração pública, baseada em critério prévio, seleciona, entre várias propostas referentes a compras, obras ou serviços, a que melhor atende ao interesse público (a fim de celebrar contrato com o responsável pela proposta mais vantajosa), tendo como princípios a legalidade, impessoalidade, moralidade, igualdade, publicidade, probidade administrativa, vinculação ao instrumento convocatório, julgamento objetivo e outros (9).

Tabela 2. Conhecimento relativo à etapa de programação e aquisição do ciclo da assistência farmacêutica (P1 e P2 - Farmacêuticos; P3 e P4 - Enfermeiros). Santa Maria-RS, 2010.

\begin{tabular}{|l|l|l|l|l|} 
& \multicolumn{1}{|c|}{ P1 } & \multicolumn{1}{c|}{ P2 } & \multicolumn{1}{c|}{ P3 } & \multicolumn{1}{c|}{ P4 } \\
\hline $\begin{array}{l}\text { Existe(m) critério(s) } \\
\text { para programação? } \\
\text { Qual(is)? }\end{array}$ & $\begin{array}{l}\text { Sim. Análise do esto- } \\
\text { que e demanda }\end{array}$ & $\begin{array}{l}\text { Sim. Demanda, saída } \\
\text { mensal, validade e } \\
\text { quantidade em estoque. }\end{array}$ & Não sabe. & Sim. Demanda da UBS. \\
\hline $\begin{array}{l}\text { Quais critérios são ob- } \\
\text { servados para a compra? }\end{array}$ & Preço & Preço/qualidade & Preço & Preço \\
\hline $\begin{array}{l}\text { Qual a forma de compra } \\
\text { de medicamentos? }\end{array}$ & Registro de preço & $\begin{array}{l}\text { Registro de preço ou } \\
\text { compra emergencial }\end{array}$ & Licitação & Licitação \\
\hline $\begin{array}{l}\text { Qual o montante finan- } \\
\text { ceiro disponibilizado? }\end{array}$ & $\begin{array}{l}\text { Não sabe ao certo, } \\
\text { acredita ser um valor } \\
\text { fixo por habitante. }\end{array}$ & $\begin{array}{l}\text { Aproximadamente 1 } \\
\text { milhão de reais. }\end{array}$ & Não & Não \\
\hline
\end{tabular}

Quando questionados em relação a essa etapa todos os participantes afirmaram que o critério utilizado para a aquisição de medicamentos é o preço, e que a compra é feita por licitação, divergindo quanto à modalidade. Um dos farmacêuticos declarou ser observada também a qualidade do produto a ser adquirido. Este mesmo farmacêutico comentou sobre a compra emergencial, em resposta à ordem judicial para medicamentos especiais. Nenhum dos entrevistados tinha certeza sobre o real valor gasto pelo município para a compra de medicamentos.

No ciclo da assistência farmacêutica o armazenamento constitui um conjunto de procedimentos técnicos e administrativos que envolvem diversas atividades, entre elas o recebimento de medicamentos, estocagem e guarda dos medicamentos, conservação e controle de estoque (4). A equipe do almoxarifado municipal deve ser especializada nos cuidados com estocagem, pois é mais fácil dispor nesse nível dos equipamentos e infraestrutura necessários à conservação dos medicamentos do que em uma UBS. Dessa forma, o almoxarifado municipal deve ser corresponsável pela estocagem nas unidades usuárias, provendo apoio técnico, informação e supervisão quanto ao processo de trabalho (6).
Os dados levantados relativos ao armazenamento estão apresentados na Tabela 3. Todos os participantes afirmaram que os medicamentos eram armazenados no Almoxarifado Central do Município, porém, divergiram quanto à qualidade das instalações. Os farmacêuticos afirmaram que o armazenamento era realizado em ambiente adequado, porém, ainda não o ideal, necessitando de melhorias no que diz respeito à climatização. Os demais profissionais apontaram problemas como insetos, pó, temperatura inadequada, local inadequado (próximo ao piso), etc. Apenas um farmacêutico soube informar sobre o tempo médio de armazenamento dos medicamentos no almoxarifado, que é de 3 (três) meses. Já nas UBSs, os medicamentos são armazenados em ordem alfabética, questão respondida corretamente por todos os entrevistados. Sobre as condições de armazenamento nas unidades, uma profissional declarou que estas não são ideais, pois em seu local de trabalho falta espaço físico, ventilação, ocorre a incidência de luz solar direta e os medicamentos ficam armazenados diretamente no solo. Os demais consideraram o armazenamento nas unidades como satisfatório. 
Tabela 3. Conhecimento relativo à etapa de armazenamento do ciclo da assistência farmacêutica (P1 e P2 - Farmacêuticos; P3 e P4 - Enfermeiros). Santa Maria-RS, 2010.

\begin{tabular}{l|l|l|l|l|} 
& \multicolumn{1}{c|}{ P1 } & \multicolumn{1}{c|}{ P2 } & \multicolumn{1}{c|}{ P3 } & \multicolumn{1}{c|}{ P4 } \\
\hline $\begin{array}{l}\text { Quais condições } \\
\text { apresentadas pelo } \\
\text { almoxarifado central? }\end{array}$ & Ótimas condições & $\begin{array}{l}\text { Próximas das ideais, } \\
\text { deve melhorar clima- } \\
\text { tização }\end{array}$ & $\begin{array}{l}\text { Não ideais, presença de } \\
\text { calor, pó }\end{array}$ & $\begin{array}{l}\text { Não ideais, presença de } \\
\text { pó, passarinho, caixa } \\
\text { no chão. }\end{array}$ \\
\hline $\begin{array}{l}\text { Qual o tempo de per- } \\
\text { manência no almoxari- } \\
\text { fado central? }\end{array}$ & Não sabe & 3 meses (em média) & Não sabe & Não sabe \\
\hline $\begin{array}{l}\text { Existe adequação de } \\
\text { espaço e localiza- } \\
\text { ção do almoxarifado } \\
\text { central? }\end{array}$ & Sim. & $\begin{array}{l}\text { Próximas das ideais } \\
\text { (pequena). }\end{array}$ & $\begin{array}{l}\text { Não, falta espaço, } \\
\text { pouca ventilação, bate } \\
\text { sol e caixas no chão. }\end{array}$ & Sim. \\
\hline
\end{tabular}

Para o descarte dos medicamentos vencidos, todos declararam conhecer o fato de que uma empresa terceirizada faz o recolhimento dos produtos quinzenalmente nas UBSs e procede o descarte através da incineração dos mesmos. Porém, uma das profissionais declarou que para produtos líquidos, o descarte é realizado no vaso sanitário, não sendo destinado ao recolhimento. Outra profissional mencionou que ao se aproximar a data do vencimento dos medicamentos, estes são enviados ao almoxarifado central para que este tome as devidas providências. Segundo os farmacêuticos, a média de vencimento é baixa, inferior a $1 \%$, sendo que isto ocorre especialmente com os produtos oriundos de amostras grátis, especialmente de medicamentos não padronizados. Os demais profissionais concordaram que a média de produtos vencidos é pequena.

$\mathrm{O}$ descarte incorreto de produtos químicos, como é o caso de medicamentos, é uma questão de saúde pública, pois resulta em contaminação do solo, dos mananciais aqüíferos e pode ser origem de utilização inadequada por parte de catadores de lixo ou outras pessoas em contato com este produto. $\mathrm{O}$ risco associados à emissão de resíduos pode variar muito, seja em função da capacidade produtiva ou dos mecanismos de segurança disponíveis (monitoração automática, segurança de armazenagem, etc.), seja em função da linha de produtos, ou seja, das características das substâncias químicas geradas $(10,11)$.

Nesta situação se faz necessária a implementação de Plano de Gerenciamento de Resíduos dos Serviços de Saúde - PGRSS, a fim de determinar os procedimentos ideias para segregar, coletar, acondicionar, tratar, armazenar e dispor dos resíduos de origem farmacológica, atividades estas regulamentadas pela Política Nacional de Resíduos Sólidos - PNRS (12, 13).

A distribuição tem início a partir de uma solicitação de medicamentos (por parte do requisitante) para o nível de distribuição envolvido, a fim de suprir necessi- dades desses medicamentos por um determinado período de tempo. Para que exista uma distribuição correta e racional deve-se garantir: rapidez na entrega, segurança e eficiência no sistema de controle. Todos os entrevistados concordaram quanto à periodicidade da distribuição, quinzenal, e quanto à forma, através de veículo da prefeitura. Os profissionais não farmacêuticos demonstraram desconhecimento e confusão de conceitos dos termos "distribuição" e "dispensação". A distribuição se refere ao suprimento de medicamentos às UBSs, em quantidade, qualidade e tempo oportuno, enquanto, dispensação diz respeito ao ato do profissional farmacêutico de proporcionar um ou mais medicamentos a um paciente, em resposta à apresentação de uma receita elaborada por um profissional autorizado, seguindo da informação e orientação do paciente em relação ao uso adequado do medicamento (4).

Um dos pontos chave para a correta condução do ciclo da assistência é a prescrição, que deve seguir critérios rígidos baseado na legislação como forma de contribuição, inclusive, para o uso racional de medicamentos. Segundo a Organização Mundial de Saúde (OMS) para que o uso racional de medicamentos ocorra, é preciso, em primeiro lugar, estabelecer a necessidade do uso do medicamento; a seguir, que se receite o medicamento apropriado, a melhor escolha, de acordo com os ditames de eficácia e segurança comprovados e aceitáveis. Além disso, é necessário que o medicamento seja prescrito adequadamente, na forma farmacêutica, doses e período de duração do tratamento; que esteja disponível de modo oportuno, a um preço acessível, e que responda sempre aos critérios de qualidade exigidos; que se dispense em condições adequadas, com a necessária orientação e responsabilidade, e, finalmente, que se cumpra o regime terapêutico já prescrito, da melhor maneira possível (14).

Os requisitos para a promoção do uso racional de medicamentos são muito complexos e envolvem uma série de variáveis, em um encadeamento lógi- 
co. Para que sejam cumpridos, devem contar com a participação de diversos atores sociais: pacientes, profissionais de saúde, legisladores, formuladores de políticas públicas, indústria, comércio, governo (15). Os entrevistados apresentaram consenso, como pode ser observado na Tabela 4, em afirmar que a maioria das prescrições era racional e trazia o nome genérico do medicamento, bem como a dosagem. Apenas um profissional declarou que, às vezes, medicamentos eram dispensados sem prescrição médica, pela utilização de critérios como o conhecimento do paciente e de seu quadro de saúde, e que este procedimento era realizado para evitar que pacientes com dor ou extremamente doentes tivessem que ficar na fila da UBS. Foram unânimes em dizer que os medicamentos faziam parte da REMUME.

Tabela 4. Conhecimento relativo à etapa de prescrição, racionalidade e comunicação do ciclo da assistência farmacêutica (P1 e P2 - Farmacêuticos; P3 e P4 - Enfermeiros). Santa Maria-RS, 2010.

\begin{tabular}{l|l|l|l|l|} 
& \multicolumn{1}{|c|}{ P1 } & \multicolumn{1}{c|}{ P2 } & \multicolumn{1}{c|}{ P3 } & \multicolumn{1}{c|}{ P4 } \\
\hline $\begin{array}{l}\text { Existe racionalidade na } \\
\text { prescrição? }\end{array}$ & Sim. & Sim. & Geralmente & Geralmente \\
\hline $\begin{array}{l}\text { Existe dispensação sem } \\
\text { prescrição? }\end{array}$ & Não & Não & Não & Não, mas há exceção \\
\hline $\begin{array}{l}\text { Existe comunicação } \\
\text { entre Prescritor/Dis- } \\
\text { pensador? }\end{array}$ & Sim & Sim & Sim & Não \\
\hline $\begin{array}{l}\text { Qual a média diária de } \\
\text { receitas dispensadas? }\end{array}$ & 50 a 100 & 70 & 65 & 90 a 100 \\
\hline $\begin{array}{l}\text { Quais as classes mais } \\
\text { prescritas? }\end{array}$ & $\begin{array}{l}\text { Analgésicos, antibió- } \\
\text { ticos e anti-hiperten- } \\
\text { sivos }\end{array}$ & $\begin{array}{l}\text { Anti-hipertensivos e } \\
\text { anti-diabéticos }\end{array}$ & $\begin{array}{l}\text { Analgésicos, anti-infla- } \\
\text { matórios e antibióticos }\end{array}$ & $\begin{array}{l}\text { Analgésicos, antibió- } \\
\text { ticos e anti-hiperten- } \\
\text { sivos }\end{array}$ \\
\hline
\end{tabular}

Apenas um dos profissionais declarou que não existe comunicação entre médico e responsável pela farmácia. O trabalho em equipe se torna pressuposto para a integralidade das ações de saúde, requerendo a construção de projeto assistencial comum para atender com qualidade as necessidades dos usuários, reconhecendo que os indivíduos não escolhem arbitrariamente viver ou trabalhar juntos, mas formam novo agrupamento diante de cada situação que se apresenta com todas as suas representações e vivências anteriores. Por isso e para isso, o trabalho deve ser integrado e não apenas acontecer no mesmo espaço, incentivado por atividades que congreguem os profissionais em torno de um objetivo maior: o bem estar do paciente (16).

Os medicamentos mais prescritos/dispensados pertencem à classe dos analgésicos, seguida pelos medicamentos antibióticos e anti-hipertensivos. Esses dados são confirmados pelo estudo realizado na Universidade Federal de Pelotas, que demonstrou que o grupo dos analgésicos e antiinflamatórios é o mais usado (26,6\%), seguido pelo dos medicamentos que atuam no sistema cardiovascular $(24,6 \%)$, em que se destacam os anti-hipertensivos $(11,0 \%)$ e os diuréticos $(6,5 \%)$. Os medicamentos que atuam no sistema endócrino e reprodutor corresponderam a $12,1 \%$ dos medicamentos utilizados; desses, 53,7\% eram contraceptivos. A prevalência de uso de contraceptivos nas mulheres em idade reprodutiva (20 a 49 anos) foi de 26,3\% (17). Quanto ao número de prescrições atendidas, este varia de 50 a 100/unidade, dependendo do tamanho da mesma e de sua área de abrangência.

Como critério exigido para dispensação de medicamentos, todos os entrevistados citaram o receituário médico e a carteira de identidade (ou outro documento de identificação com foto) e declararam que orientam sobre a utilização dos medicamentos. Farmacêuticos declararam que os pacientes recebem bem a informação e que agradecem. Os demais profissionais declararam que os pacientes muitas vezes nem prestam atenção, mas que a maioria tem interesse. Merece destaque a utilização de técnicas de abordagem efetivas para a transmissão da informação em saúde, especialmente em termos da escolha da linguagem adequada. A comunicação envolve mais que simples palavras, utilizando-as na escrita ou falada e empregando ainda signos e sinais não verbais. Para os profissionais da saúde em geral, a comunicação pode ser definida como a troca de informações ou mensagens com o paciente. As bases da atenção farmacêutica determinam o paciente como foco principal de ação, tornando a utilização das técnicas de comunicação de fundamental importância para o farmacêutico (18). 
O seguimento farmacoterapêutico na farmácia básica não é efetivo em nenhuma das unidades visitadas, como pode ser evidenciado na Tabela 5, sendo que todos os responsáveis declararam não haver controle e avaliação do uso de medicamentos após a dispensação. Exceção a este fato é o programa HiperDia (Programa de acompanhamento de pacientes com diabetes mellitus e/ou hipertensão arterial), citado por todos os profissionais, que realiza um acompanhamento mais efetivo mediante o cadastro de pacientes para dispensação de medicamentos. O HiperDia surge para reorganizar os serviços, para o oferecimento de uma atenção continuada e qualificada aos portadores de diabetes mellitus e/ou hipertensão arterial. Foi priorizado por este plano a confirmação de casos suspeitos; a elaboração de protocolos clínicos e treinamentos dos profissionais de saúde; a garantia da distribuição gratuita de medicamentos anti-hipertensivos, hipoglicemiantes orais e insulina NPH. O HiperDia também realiza o monitoramento dos pacientes captados neste plano e gera informações para a aquisição, dispensação e distribuição de medicamentos, de forma regular e contínua, a todos os pacientes cadastrados no programa (19).

Tabela 5. Conhecimento relativo ao controle e avaliação do uso de medicamentos (P1 e P2 - Farmacêuticos; P3 e P4 - Enfermeiros). Santa Maria-RS, 2010.

\begin{tabular}{|c|c|c|c|c|}
\hline & P1 & P2 & P3 & P4 \\
\hline $\begin{array}{l}\text { Existe controle e } \\
\text { avaliação do uso dos } \\
\text { medicamentos? Quais? }\end{array}$ & Não & $\begin{array}{l}\text { Não (exceção para o } \\
\text { programa hiperdia) }\end{array}$ & $\begin{array}{l}\text { Não (exceção para o } \\
\text { programa hiperdia) }\end{array}$ & Não \\
\hline $\begin{array}{l}\text { A UBS participa de } \\
\text { algum programa? }\end{array}$ & Hiperdia & Hiperdia. & Não & $\begin{array}{l}\text { Hiperdia, suplemen- } \\
\text { tação de ferro com } \\
\text { gestantes e puérperas e } \\
\text { pós- aborto }\end{array}$ \\
\hline $\begin{array}{l}\text { Existe demanda repri- } \\
\text { mida? Qual? }\end{array}$ & $\begin{array}{l}\text { Sim, informatização } \\
\text { das UBSs }\end{array}$ & Não & $\begin{array}{l}\text { Sim, medicamentos, } \\
\text { espaço, atuação junto } \\
\text { ao paciente }\end{array}$ & $\begin{array}{l}\text { Sim, orientação sobre } \\
\text { medicamentos, con- } \\
\text { trole e avaliação dos } \\
\text { medicamentos }\end{array}$ \\
\hline
\end{tabular}

Uma unidade avaliada não participa do Hiperdia nem de outros programas como suplementação de ferro em gestantes e puérperas e do grupo pós-aborto. A participação do farmacêutico deve ser mais efetiva junto aos grupos, uma vez que pode contribuir de maneira valiosa para à melhora clínica, econômica e humanística no cuidado com o paciente, tanto participando de grupos de apoio ao paciente, como trabalhando em conjunto com as equipes de saúde das UBSs. As intervenções farmacêuticas incluem identificação de possíveis interações medicamentosas, ajuste da dose, troca ou suspensão de um dos medicamentos e alteração dos horários (20).

Quanto às necessidades do local foram apontados como demanda reprimida das UBSs a informatização da unidade para melhoria do controle de dispensação de medicamentos e de estoque (apontado por farmacêuticos), uma maior atuação junto ao paciente, maior espaço físico, ampliação da oferta de medicamentos, em número e diversidade (apontado por não farmacêuticos).

\section{CONCLUSÕES}

Este estudo contribuiu para destacar o potencial de atuação do profissional farmacêutico nas UBSs, para a garantia da promoção da saúde, do uso racional de medicamentos, da educação em saúde, orientação e acompanhamento do medicamento, entre outras possíveis contribuições. Pode-se afirmar que o conhecimento sobre o medicamento é um diferencial necessário às práticas relacionadas à utilização de medicamentos dentro das UBSs.

A insatisfatória participação do farmacêutico nas equipes de saúde gera uma situação perturbadora, pois é o profissional farmacêutico o co-responsável pela assistência farmacêutica em toda sua extensão, desempenhando papel fundamental em relação ao medicamento, ficando assim as UBSs sem um sistema organizacional de assistência farmacêutica completo, deixando o medicamento e os cuidados relacionados ao seu uso desordenados. Somente através do cumprimento fiel a todas as etapas do ciclo da assistência farmacêutica, poderá se garantir um acesso qualificado ao medicamento. 


\section{REFERÊNCIAS}

1. Consenso Brasileiro de Atenção Farmacêutica. Propostas. Atenção Farmacêutica no Brasil: "Trilhando Caminhos”. Brasília: Organização Pan-Americana de Saúde, 2002.

2. Organizacion Mundial de La Salud (OMS). El papel del farmacéutico em el sistema de atención de salud. Nueva Delhi, OMS, 1988.

3. Coradi AEP. A importância do farmacêutico no ciclo da Assistência Farmacêutica. Arquivos Brasileiros de Ciências da Saúde. 2012; 37(2):62-4.

4. Brasil. Ministério da Saúde. Departamento de Atenção Básica. Assistência farmacêutica na atenção básica: Instruções técnicas para a sua organização. Brasília, 2001.

5. Pepe VLE. O formulário terapêutico nacional, os protocolos clínicos e diretrizes terapêuticas: extensões da seleção de medicamentos. Ministério da Saúde, 2009.

6. Marin N, Luiza VL, Castro CGSO, Santos SM. Assistência farmacêutica para gerentes municipais. Rio de Janeiro: OPAS/OMS, 2003.

7. Brasil. Secretaria de Ciências Tecnológicas e Insumos Estratégicos. Assistência farmacêutica na atenção básica: instruções técnicas para sua organização. 2 ed. Brasília, DF, 2006.

8. Dalenogare MM. Assistência Farmacêutica no programa de controle de tuberculose: planejamento versus execução da programação de medicamentos [tese]. Porto Alegre (RS): Universidade Federal do Rio Grande do Sul, 2009.

9. Agência Nacional de Vigilância Sanitária (ANVISA). Vigilância sanitária e licitação pública. Brasilía, 2003.

10. Linninger AA, Chakraborty A, Colberg RD. Synthesis and optimization of waste treatment flowsheets. Comp. Chem. Eng. 1999, 23: 1415-25.

11. Linninger AA, Chakraborty A, Colberg RD Planning of waste reduction strategies under uncertainty. Comp. Chem. Eng. 2000. 24:1043-48.
12. Brasil. Congresso Nacional. Lei $n^{\circ} .12 .305$, de 02 de agosto de 2010. Institui a Política Nacional de Resíduos Sólidos; altera a Lei no 9.605, de 12 de fevereiro de 1998; e dá outras providências. Diário da União, Poder Executivo, Brasília, DF, 03 de ago. de 2010. Seção 1. p. 2. Disponível em: <http://www.planalto.gov. br/ccivil_03/_ato2007- 2010/2010/lei/112305.htm >. Acesso em 15 de fevereiro de 2013.

13. Reis SC, Reis, MC, Vasconcelos V, Jordão E. Elementos para uma abordagem sistemática da gestão de resíduos farmacológicos à luz da política nacional de resíduos sólidos (PNRS) Revista Brasileira de Políticas Públicas. 2012, 2(2):71-87.

14. Organização Mundial da Saúde (OMS). The rational use of drugs:report of the conference of experts. Nairobi, 1985. Jul 2529. Geneva: WHO; 1987.

15. Aquino DS. Por que o uso racional de medicamentos deve ser uma prioridade? Cien. Saúde Colet. 2008, 13:733-736.

16. Pedroso JIS, Teles JBM. Consenso e diferenças em equipes do Programa Saúde da Família. Rev. Saúde Pública. 2001, 35(Supl.3):303-11.

17. Bertoldi AD, Barros AJD, Hallal P, Lima, RC Utilização de medicamentos em adultos: prevalência e determinantes individuais. Rev. Saúde Pública.2004, 38 (Supl.2):228-38.

18. Cordeiro $\mathrm{BC}$, Leite $\mathrm{SN}$. O farmacêutico na atenção à saúde. 2. ed. Itajaí: Univali; 2008.

19. Chazani AC, Perez EA. Avaliação da implementação do sistema informatizado de cadastramento e acompanhamento de hipertensos e diabéticos (HIPERDIA) nos municípios do estado do Rio de Janeiro. Rev. APS. 2008, 11(Supl.1): 10-16.

20. Mahumud SDP, Martinbiancho JK, Zuckermann J, Jacoby TS, Santos L, Silva D. Assistência farmacêutica: ações de apoio à qualidade assistencial. Rev. Infarma. 2006, 18(7/8). 